\title{
Local Changes in Gravity Resulting From Deformation
}

\author{
J. B. WALSH \\ Department of Earth and Planetary Sciences, Massachusetts Institute of Technology. Cambridge, Massachusetts 02139 \\ J. R. RICE \\ Division of Engineering, Brown University, Providence, Rhode Island 02912

\begin{abstract}
The horizontal and vertical components of gravity change when tectonic stresses deform the earth because mass is redistributed relative to the gravity meter. We analyze the change in gravity resulting from deformation in a homogeneous elastic half-space. We derive expressions in closed form which give the change in horizontal and vertical components of gravity measured at the surface for any specified distribution of dislocations at depth. For example, the change in the vertical component of gravity observed by a gravity meter fixed in space above an infinitely long thrust fault is found to be proportional to the local change in height, whereas the change due to a spherically symmetric source of dilatation is zero. Analysis of the change in the horizontal component shows that error in measurements of uplift resulting from changes in level is negligible for these sources.
\end{abstract}

\section{INTRODUCTION}

Gravity changes determined from surveys made before and after the Alaskan (1964) earthquake [Barnes, 1966], the San Fernando (1971) earthquake [Oliver et al., 1972], and the Inangahua (New Zealand, 1968) earthquake $[H$ unt, 1970] were found to be proportional to local changes in elevation. The close correlation between uplift and gravity change suggests that the tectonic straining which produced the uplift also altered the local gravity field. Changes in elevation and horizontal surface displacements have become an important source of information about tectonic processes occurring before and during earthquakes, and sophisticated analyses of these data are now possible. Clearly, developing similar techniques for analyzing changes in the gravity field is of great practical importance.

Whitcomb [1976] suggests that measurements of uplift may themselves be affected by changes in gravity. Redistribution of mass due to tectonic straining changes the horizontal component of gravity as well as the vertical component. Changes in the horizontal component cause local changes in level, and although these are small, Whitcomb suggests that they become appreciable when integrated over long traverses.

Deriving the volume integral giving the change in gravity resulting from a specified displacement field is straightforward. However, displacement fields produced by even the simple sources used to simulate faults or dilatancy are too complicated to permit direct computation of gravity changes except numerically. As a consequence, simple models have been used to get approximate results. In these models [Whitcomb, 1976; Ruff et al., 1976], uplift is simulated by uniformly deforming a cylinder imbedded in the earth's surface; deformation in the cylinder is decoupled from the surrounding rock, which is unstrained. The deformation field in these studies is highly idealized, and so application to real tectonic events is uncertain until results from more realistic models are available for comparison.

We have developed a technique involving, in effect, the reciprocal theorem, which allows more sophisticated models of tectonic events to be analyzed. We assume that the earthquake covers a sufficiently small volume that the earth can be considered to be a half-space. We assume further that the rock

Copyright $\odot 1979$ by the American Geophysical Union.

Paper number 8B0920.

$0148-0227 / 79 / 008 \mathrm{~B}-0920 \$ 01.00$ is linearly elastic, homogeneous, and isotropic. Deformation of the half-space occurs as a consequence of some as yet unspecified displacement source at depth. After developing general equations below, we use them to calculate the gravity changes due to a center of dilatation and to a long thrust fault. The model and the sources are thus the same as those used in traditional analyses of surface displacements resulting from faulting and dilatancy.

\section{ANALYSIS}

\section{Vertical and Horizontal Components of Gravity}

The gravimeter is represented in Figure 1 by the point mass suspended above the elastic half-space. The material in the half-space exerts a gravitational pull on the point mass, and so the vertical force $P_{z}$ is needed to keep it in equilibrium. The gravitational force vector is, in general, not vertical, and so horizontal forces, represented by $P_{r}$ in Figure 1, may also be needed. The gravitational pull of the point mass generates stresses in the half-space; the stresses acting along an arbitrary surface $s$ are denoted by $\sigma_{i}$ in the figure. The stress associated with self-gravitation of the half-space is neglected, a standard assumption in the theoretical faulting calculations referred to above.

The differential $d E$ in energy (elastic and gravitational) of the system when the point mass is displaced vertically by $d c_{z}$ and increments $d u_{t}$ of relative displacement are imposed on $s$ is

$$
d E=P_{z} d c_{z}+\int_{8} \sigma_{i j} d u_{i} d s_{j}
$$

(Here the sign convention is such that if the sides of $s$ are labeled positive and negative and if $u_{t}$ is defined as the displacement on the positive side minus that on the negative side, then the directed area element $d s_{\text {, }}$ points from positive to negative.) Now consider the following two processes, in both of which the mass is moved a small vertical distance $\Delta c_{z}$ and the displacements $u_{t}$ are imposed on $s$. For the first process we move the point mass by $\Delta c_{z}$, thereby changing $\sigma_{i j}$ to $\sigma_{i j}+$ $\left(\partial \sigma_{i j} / \partial c_{z}\right)_{u_{t}} \Delta c_{z}$, and then we impose the displacements $u_{t}$ on $s$. The change in energy, to second order in $\Delta c_{z}$ and $u_{l}$, is

$$
\begin{aligned}
\Delta E=P_{z} \Delta c_{z}+ & 1\left(\partial P_{z} / \partial c_{z}\right)_{u_{l}}\left(\Delta c_{z}\right)^{2} \\
& +\int_{\delta}\left[\sigma_{i \jmath}+\left(\partial \sigma_{l \jmath} / \partial c_{z}\right) u_{i} \Delta c_{z}+L \tilde{\sigma}_{i \jmath}\right] u_{l} d s_{\jmath}
\end{aligned}
$$

where $\tilde{\sigma}_{i j}$ is the change in stress due to introducing $u_{t}$ with $c_{z}$ 


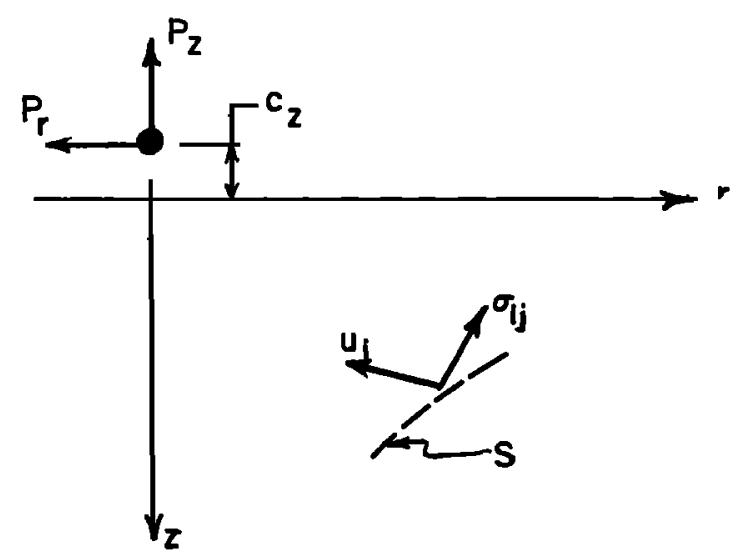

Fig. 1. Forces $P_{2}$ and $P_{r}$ hold a gravitating point mass (solid circle) above an elastic half-space, producing stress $\sigma_{i j}$ on surface $s$. Equation (1) gives the change in energy when the point mass is moved a small vertical distance $d c_{2}$ and the surface $s$ is allowed to undergo relative displacement $d u_{i}$.

fixed. For the second process we impose the relative displacements $u_{t}$ on $s$, thereby changing $P_{z}$ to $\Delta P_{z}$, and then we move the point mass by $\Delta c_{z}$. The energy change (again to second order) is

$$
\begin{aligned}
\Delta E=\int_{s}\left(\sigma_{i j}+l \tilde{\sigma}_{i j}\right) u_{l} d s_{j}+\left(P_{z}\right. & \left.+\Delta P_{z}\right) \Delta c_{z} \\
& +\frac{1}{2}\left(\partial P_{z} / \partial c_{z}\right)_{u_{i}}\left(\Delta c_{z}\right)^{2}
\end{aligned}
$$

The expressions for $\Delta E$ in (2) and (3) must be identical, and so the change $\Delta P_{z}$ in vertical force on the point mass due to $u_{t}$ is

$$
\Delta P_{z}=\int_{s}\left(\partial \sigma_{i j} / \partial c_{z}\right)_{u_{i}} u_{i} d s_{j}
$$

The force $P_{z}$ is $m g_{z}$, where $g_{z}$ is the vertical component of gravitational acceleration, and so (4) can be written

$$
\Delta g_{z}=(1 / m) \int_{s}\left(\partial \sigma_{i j} / \partial c_{z}\right)_{u_{i}} u_{i} d s_{j}
$$

or in simpler notation,

$$
\Delta g_{z}=\int_{8} s_{l j}^{2} u_{l} d s_{j}
$$

where

$$
S_{l j}^{2}=(1 / m)\left(\partial \sigma_{i j} / \partial c_{z}\right)_{u_{l}}
$$

Note in (5) that the change in the vertical component of gravity due to any dislocation $u$ in the half-space can be found once we evaluate $S_{i j}{ }^{2}$. This term is simply the change in stress $\sigma_{i j}$ in a dislocation-free half-space due to moving the reference mass a small vertical distance.

The change $\Delta g_{r}$ in a horizontal component of gravity is found following the same procedure, with the result

$$
\Delta g_{r}=\int_{s} S_{i j} u_{i} d s_{j}
$$

where

$$
S_{i j}{ }^{r}=(1 / m)\left(\partial \sigma_{i j} / \partial c_{r}\right)_{u_{i}}
$$

Here, we must find the change in $\sigma_{i j}$ due to moving the reference mass a small horizontal distance (in a direction opposite to that chosen as positive for $g_{r}$ ). Notice that the stresses resulting from moving the reference mass must be calculated only once; once they are known, the change in gravity resulting from any specified distribution of displacement at depth can be found from (5) and (6).
The stress field $\sigma_{i j}$ due to a gravitating point mass, at height $c$ above the surface of an elastic half-space (as in Figure 1), is derived in the appendix. The results are

$$
\begin{aligned}
& \sigma_{z z}=G m \rho z(z+c) / R^{a} \\
& \sigma_{r z}=G m \rho r z / R^{s} \\
& \sigma_{\theta \theta}=-G m \rho z / R(R+z+c) \\
& \sigma_{r r}=G m \rho\left[z / R(R+z+c)-z(z+c) / R^{3}\right]
\end{aligned}
$$

where $R^{2}=r^{2}+(z+c)^{2}, G$ is the gravitational constant, and $\rho$ is density. Note in (7) that $\sigma_{u}=\sigma_{r r}+\sigma_{\theta \theta}+\sigma_{z z}=0$, that is, the point mass induces no dilatation in the half-space. Expressions for $S_{\ell j}{ }^{z}$, defined by (5), for a point fixed in space very near the earth's surface $(c=0)$ are found from $(7)$ to be

$$
\begin{aligned}
& S_{r r}{ }^{2}=\left(G \rho z / R^{3}\right)\left(1-3 r^{2} / R_{2}\right) \\
& S_{\theta \theta}^{z}=G \rho z / R^{s} \\
& S_{z z}{ }^{z}=\left(G \rho z / R^{8}\right)\left(1-3 z^{2} / R^{2}\right) \\
& S_{r z}^{z}=-3 G \rho r z^{2} / R^{b} \\
& S_{u u^{2}}=S_{r r}^{z}+S_{\theta \theta}^{z}+S_{z z}{ }^{2}=0
\end{aligned}
$$

To calculate $S_{i j^{x}}$, we first express $\sigma_{i j}$ in (7) in rectangular coordinates as follows:

$$
\begin{gathered}
\left(\sigma_{x z,}, \sigma_{y z}\right)=(x, y)\left(\sigma_{r z} / r\right) \\
\left(\sigma_{x x}, \sigma_{x y}, \sigma_{y y}\right)=(1,0,1) \sigma_{\theta \theta}+\left(x^{2}, x y, y^{2}\right)\left(\sigma_{r r}-\sigma_{\theta \theta}\right) / r^{2}
\end{gathered}
$$

The components of $S_{l j}{ }^{x}$ in (6), corresponding to changes in the horizontal component of gravity, are given by $(1 / m)\left(\partial \sigma_{i j} / \partial x\right)$ because of the translational invariance of $\sigma_{l j}$. We find that the components of $S_{i j}{ }^{x}$ are

$$
\begin{aligned}
& S_{x x}{ }^{x}=\left(G \rho z x / R^{3}\right)\left[\frac{3 z x^{2}}{R^{2} r^{2}}+\left(3-4 \frac{x^{2}}{r^{2}}\right) \frac{2 R+z}{(R+z)^{2}}\right] \\
& S_{y y}{ }^{2}=\left(G \rho z x / R^{3}\right)\left[\frac{3 z y^{2}}{R^{2} r^{2}}+\left(1-4 \frac{y^{2}}{r^{2}}\right) \frac{2 R+z}{(R+z)^{2}}\right] \\
& S_{z z}{ }^{x}=-3 G \rho z^{2} x / R^{5} \\
& S_{x y}^{x}=\left(G \rho z y / R^{9}\right)\left[\frac{3 z x^{2}}{R^{2} r^{2}}+\left(1-4 \frac{x^{2}}{r^{2}}\right) \frac{2 R+z}{(R+z)^{2}}\right] \\
& S_{x z}{ }^{x}=\left(G \rho z / R^{3}\right)\left(1-3 x^{2} / R^{2}\right) \\
& S_{y z}{ }^{x}=-3 G \rho x y z / R^{5} \\
& S_{l t} x=S_{x x} x+S_{y y} x+S_{z z} x=0
\end{aligned}
$$

Corresponding expressions for $S_{i j}^{y}$ can be found by exchanging $x$ and $y$ in each equation in (10).

\section{Change in Potential and Error in Uplift}

We see from (5) and (6) that the change $\Delta g_{k}$ in any specified component of the gravity vector is

$$
\Delta \boldsymbol{g}_{k}=(1 / m) \int_{a}\left(\partial \sigma_{i j} / \partial c_{k}\right) u_{i} d s_{\jmath}
$$

or, equivalently,

$$
\Delta g_{k}=\left(\partial / \partial c_{k}\right)\left[\int_{\theta}\left(\sigma_{t \jmath} / m\right) u_{t} d s_{j}\right]
$$

The change $\Delta g_{k}$ in a component of gravity is defined in terms of the change $\Delta V$ in potential by the relation 


$$
\Delta g_{k}=\left(\partial / \partial c_{k}\right)(\Delta V)
$$

Comparing (11) and (12), we see that the change $\Delta V$ in potential is given by

$$
\Delta V=\int_{s}\left(\sigma_{l j} / m\right) u_{i} d s_{j}
$$

where $\sigma_{t j}$ is given by (7).

As proposed by Whitcomb [1976], changes in the horizontal component of gravity cause error in measurements of uplift if the resulting change in level is not corrected. Let us assume for simplicity that the leveling route is along the $x$ axis. The change $\Delta \alpha$ in level resulting from a change $\Delta g_{x}$ in the horizontal component of gravity is then

$$
\Delta \alpha=\Delta g_{x} / g_{z}
$$

where $g_{z}$ is the vertical component of gravity. Changes in the vertical component are small relative to $g_{z}$, and so we can assume that $g_{z}$ is constant. The error $e$ in uplift for a traverse which starts a great distance from the source is

$$
e=\int_{-\infty}^{x}\left(\Delta g_{x} / g_{x}\right) d x
$$

Substituting (12) (for $c_{k}$ in the $x$ direction) into (15), we find

$$
e=-\int_{-\infty}^{\pi}(\partial \Delta V / \partial x)\left(1 / g_{z}\right) d x=-\Delta V / g_{z}
$$

or, from (13),

$$
e=-\int_{s}\left(\sigma_{i j} / m g_{z}\right) u_{l} d s_{j}
$$

Clearly, (16) is valid whatever route is used, so long as the traverse begins where the change in potential is zero.

\section{EXAMPLES}

\section{Vertical Component of Gravity}

We see from the expression for $S_{u}^{z}$ in (8) that the change in pressure due to moving the point mass is zero everywhere in the half-space. Therefore, from (5), the change $\Delta g_{z}$ in gravity resulting from any spherically symmetric dilatational source, as observed by a gravity meter fixed in space, is

$$
\Delta g_{z}=0
$$

Of course, this result is for a dilatational source in dry rock. Rock is usually saturated with water in situ, and gravity changes due to fluid migration can be expected to occur.

Consider now a very long thrust fault parallel to the $y$ axis as in Figure 2. We see from (5) that we must find the shear component $S_{s n}{ }^{2}$ on the fault surface $s$. To find $S_{s n}{ }^{2}$, we first transform expressions for $S_{\ell j^{z}}$ given in (8) into Cartesian coordinates; the resulting expressions relevant to the problem here are

$$
\begin{aligned}
& S_{x x}^{z}=\left(G_{\rho z} / R^{3}\right)\left(1-3 x^{2} / R^{2}\right) \\
& S_{z z}{ }^{2}=\left(G \rho z / R^{3}\right)\left(1-3 z^{2} / R^{2}\right) \\
& S_{x z}{ }^{2}=-3 G \rho x z^{2} / R^{5}
\end{aligned}
$$

The expression for $S_{s n}{ }^{2}$ for an element of fault surface dipping at an angle $\beta$ from the horizontal is found from (18) to be

$$
S_{s n}{ }^{2}=\left(3 G \rho z / 2 R^{6}\right)\left[2 x z \cos 2 \beta-\left(x^{2}-z^{2}\right) \sin 2 \beta\right]
$$

The change in gravity $\Delta g_{2}$ on the surface is therefore

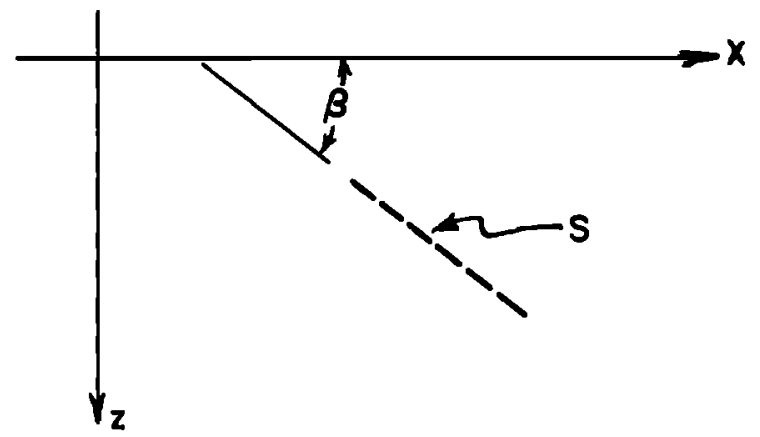

Fig. 2. Surface $s$ is a thrust fault in a half-space parallel to the $y$ axis and dipping at angle $\beta$.

$$
\Delta g_{2}=\int_{g} S_{8 n}^{2} u d s
$$

where $u$ is slip displacement, which in general, may vary along $s$, and $S_{\mathrm{an}}{ }^{z}$ is given by (19). We assume that $u$ and $\beta$ are uniform in the $y$ direction. Carrying out the integration over $y$, we find

$$
\Delta g_{z}=2 G_{\rho} \int_{s} \frac{z}{\left(x^{2}+z^{2}\right)^{2}}\left[2 x z \cos 2 \beta-\left(x^{2}-z^{2}\right) \sin 2 \beta\right] u d s
$$

For comparison we calculate the uplift resulting from slip on a long fault parallel to the $y$ axis. We find following the analysis given in the appendix that the uplift $h$ resulting from slip which is uniform in the $y$ direction is

$h=(1 / \pi) \int_{a} \frac{z}{\left(x^{2}+z^{2}\right)^{2}}\left[2 x z \cos 2 \beta-\left(x^{2}-z^{2}\right) \sin 2 \beta\right] u d s$

Combining (20) and (21), we find that the change in the vertical component of gravity is uniquely related to the local uplift by

$$
\Delta g_{z}=2 \pi G \rho h
$$

for any very long fault where the slip distribution is uniform along the direction of strike.

\section{Error in Uplift}

We see that error in uplift can be calculated from (16) using expressions for $\sigma_{i j}$ from (7). As a first example, consider a spherically symmetric source of dilatation. Because $\sigma_{u}$ in (7) vanishes, the error in uplift is zero for this source.

Next consider a thrust fault which, for simplicity, is parallel to the $x-y$ plane with displacement $u$ in the $x$ direction. Uplift is small for this configuration and $\Delta g_{x}$ is large, thereby producing a larger relative error in uplift than for other fault configurations. Error in uplift is calculated from (16) using the expression for $\sigma_{x z}$ from (7). We find that the error $e$ for a fault at depth $d$ is

$$
e=-(3 / 4 \pi)\left(\rho / \rho_{E}\right)\left(d / R_{E}\right) \int_{\theta}\left(x / R^{3}\right) u(x, y) d x d y
$$

where $\rho_{E}\left(=5.5 \mathrm{~g} / \mathrm{cm}^{3}\right)$ and $R_{E}$ are the average density and radius of the earth, and we have introduced the approximate relation $g_{z}=(4 \pi / 3) \rho_{E} G R_{E}$.

For comparison, we calculate the actual uplift $h$ using an application of the reciprocal theorem in three dimensions similar to the two-dimensional form in the appendix. We obtain 


$$
h=\int_{a}\left(\hat{\sigma}_{\ell \jmath} / Q\right) u_{\imath} d s_{\jmath}
$$

where $\hat{\sigma}_{i j}$ is the stress field induced in an elastic half-space by a vertical point force $Q$ at the origin [Timoshenko and Goodier, 1951, p. 364]:

$$
\begin{aligned}
& \hat{\sigma}_{z z}=-(3 Q / 2 \pi) z^{3} / R^{6} \\
& \hat{\sigma}_{r r}=-(Q / 2 \pi)\left\{3 r^{2} z / R^{5}-[\mu /(\lambda+\mu)] / R(R+z)\right\} \\
& \hat{\sigma}_{\theta \theta}=-[Q \mu / 2 \pi(\lambda+\mu)]\left[1 / R(R+z)-z / R^{3}\right] \\
& \hat{\sigma}_{r z}=-(3 Q / 2 \pi) r z^{2} / R^{5}
\end{aligned}
$$

Uplift $h$ found from (24) and (25) for the horizontal fault is

$$
h=-(3 / 2 \pi) d^{2} \int_{s}\left(x / R^{3}\right) u(x, y) d x d y
$$

Comparing expressions for $e$ and $h$ above, we see that the relative error $(e / h)$ contains the factor $\left(d / R_{E}\right)$, and so errors in uplift are negligible for events of practical interest. For example, if the fault is infinitely long in the $y$ direction and extends from $x=l_{1}$ to $x=l_{2}$, we find, for uniform displacement $u$, that

$$
e=-\frac{3 u}{4 \pi} \frac{\rho}{\rho_{E}} \frac{d}{R_{E}} \log \left(\frac{l_{2}^{2}+d^{2}}{l_{1}^{2}+d^{2}}\right)
$$

whereas

$$
h=-\frac{u}{\pi} \frac{d^{2}\left(l_{2}^{2}-l_{1}^{2}\right)}{\left(l_{2}^{2}+d^{2}\right)\left(l_{1}^{2}+d^{2}\right)}
$$

Thus if the observation point is directly above one end of the fault $\left(l_{1}=0\right)$ and the fault width is $W\left(=l_{2}\right)$,

$$
\frac{e}{h}=\frac{3 d}{4 R_{E}}\left(1+\frac{d^{2}}{W^{2}}\right) \log \left(\frac{W^{2}}{d^{2}}+1\right)
$$

which is always negligible.

\section{Discussion}

We develop in the analysis above algebraic expressions which can be used to calculate the changes in gravity due to any specified sources of displacement at depth. These theoretical results are applied to two types of sources, a center of dilatation and a very long thrust fault, and the changes in both the vertical and horizontal components are examined. The change in the horizontal component is found to be sufficiently small that the error in measurements of uplift due to errors in level is negligible. Whitcomb [1976] found that error in level led to appreciable errors in uplift in his model. The lack of agreement in our conclusions apparently is due to the differences in the deformation fields. All density change occurs in a thin layer near the surface in Whitcomb's model, and so gravity changes are much greater than those in the model analyzed here, where deformation is not confined to bounded regions.

We find that the change in the vertical component of gravity due to deformation alone is zero for any purely dilatational source and proportional to the local uplift for a very long thrust fault of any dip (see (17) and (22)). In our analysis the gravimeter is assumed to be fixed in space in order to eliminate the free air correction from the calculations. In actual field surveys the gravimeter is positioned on the earth's surface, which moves, and so the free air correction must be included. Therefore according to our calculations, the change in gravity which will be observed for a dilatational source is

$$
\Delta g=\Delta g_{F A}
$$

and, for a very long thrust fault,

$$
\Delta g=\Delta g_{F A}+2 \pi G \rho h
$$

where $\Delta g_{F A}$ is the free air correction $(\simeq-0.309 \mathrm{mgal} / \mathrm{m})$ and $h$ is positive for uplift. Note that (31) gives a value which is the same as if material were taken from regions of subsidence and piled in regions of uplift. The change in gravity for this case must be equal to the sum of Bouguer and free air corrections; this is just the value given by (31).

A comparison of the relative magnitudes of the terms in (31) can be made by noting that, to a reasonable approximation,

$$
\Delta g_{F A}=-(8 \pi / 3) G \rho_{E} h
$$

Combining (31) and (32) gives

$$
\Delta g_{z}=\Delta g_{F A}\left[1-\left(3 \rho / 4 \rho_{E}\right)\right]
$$

We find for $\rho=2.6 \mathrm{~g} / \mathrm{cm}^{2}$ that

$$
\Delta g_{z}=0.65 \Delta g_{F A}=-0.20 h \mathrm{mgal} / \mathrm{m}
$$

Rundle [1978] calculated numerically the gravity change due to a point source of dilatation and for an infinitely long buried thrust fault dipping at $10^{\circ}$ in a material where $\lambda=\mu$. His results for the dilatational source agree with (30). He finds for the thrust fault that gravity changes are proportional to the local uplift, as in (31). The constant of proportionality is approximately the same as our value.

In the three earthquakes for which data are available, local changes in gravity were found to be approximately proportional to the changes in height. We see in (31) that the analysis here predicts that uplift and gravity change should be linearly related, in agreement with the observation. Further, the constant of proportionality calculated from our analysis agrees well with the value derived from field data. The observed value (see Table 1) is approximately $-0.2 \mathrm{mgal} / \mathrm{m}$.

One possible cause of small discrepancies is that (31), which applies to very long faults, is not strictly applicable to the faults being considered, which were more nearly equidimensional. We studied the effect of finite fault length in an approximate way by analyzing the change in gravity for a small, very deep fault. We found that a correction factor must be applied to (31), which shifts the theoretical changes in gravity. The correction factor depends upon the location of the gravity meter, however, and so one cannot be sure that the agreement between theory and observation is improved without considering each station individually.

The relationship between gravity change and uplift in some earthquakes is not as simple as in the earthquakes that we use for illustration here. Gravity changes and elevation changes were observed before and after the Matsushiro (1966) earthquake swarm [Nur, 1974; Kisslinger, 1975; Stuart and Johnston, 1975] and the Heicheng (1975) and Tangshan (1976) earthquakes [Chen et al., 1977]. These authors suggest that the migration of fluids such as water and magma occurred in

TABLE 1. Observed Changes in Gravity and Uplift

\begin{tabular}{lc}
\hline \multicolumn{1}{c}{ Reference } & $\Delta g / h, \mathrm{mgal} / \mathrm{m}$ \\
\hline Alaska, 1964 [Barnes, 1966] & -0.197 \\
Inangahua, 1968* [Hunt, 1970] & $-0.15 ;-0.20$ \\
San Fernando, 1971 [Oliver et al., 1972] & -0.215 \\
\hline
\end{tabular}

*Only two stations occupied. 


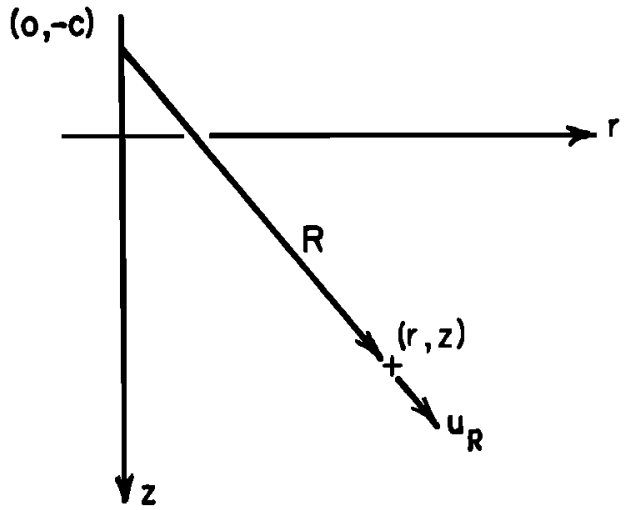

Fig. 3. The radius vector $R$ extends from a point mass located at $(0,-c)$ in an infinite medium to an arbitrary point $(r, z)$. Body forces due to the gravitating point mass produce displacements $u_{R}$ symmetric about $(0,-c)$.

conjunction with these events. The changes in the gravity field, which is the superposition of the changes due to fluid migration and the changes due to elastic deformation that are analyzed above, are complex functions of time, and so we have not attempted to interpret field data from these events.

\section{APPENDIX}

\section{Change in Gravity}

As described in the text, we derive expressions for $\sigma_{l j}$ by first finding the stress field in an unbounded elastic body induced by a gravitating point mass $m$. Suppose that the point mass is located at $(0,0,-c)$, as in Figure 3 . The body force $F_{R}$ per unit volume due to the mass is directed along $R$ and has the value

$$
F_{R}=-G m \rho / R^{2}
$$

where $R^{2}=r^{2}+(z+c)^{2}$. Because of symmetry, the only component of deformation is $u_{R}$, and so the equilibrium equations in spherical coordinates [e.g., Fung, 1965] reduce to

$(\lambda+2 \mu) \frac{\partial^{2} u_{R}}{\partial R^{2}}+2(\lambda+2 \mu)\left(\frac{1}{F} \frac{\partial u_{R}}{\partial R}-\frac{u_{R}}{R^{\prime}}\right)=G m \rho / R^{2}$

where $\lambda$ and $\mu$ are the Lamé moduli. Solving (A2), we find that displacement is uniform, with the value

$$
u_{R}=-(G m \rho) / 2(\lambda+2 \mu)
$$

Stress components corresponding to $u_{R}$ in (A3) are

$$
\begin{aligned}
& \sigma_{z z}=-[G m \rho / R(\lambda+2 \mu)]\left(\lambda+\mu r^{2} / R^{2}\right) \\
& \sigma_{r r}=-[G m \rho / R(\lambda+2 \mu)]\left(\lambda+\mu z^{\prime 2} / R^{2}\right) \\
& \sigma_{\theta \theta}=-(G m \rho / R)[(\lambda+\mu) /(\lambda+2 \mu)] \\
& \sigma_{r z}=(G m \rho)(\mu / \lambda+2 \mu)\left(r z^{\prime} / R^{3}\right)
\end{aligned}
$$

where $z^{\prime}=z+c$. Note in (A4) that vertical and horizontal 'tractions' are present on the plane $z=0$ :

$$
\begin{aligned}
& \sigma_{z z}=-[G m \rho / R(\lambda+2 \mu)]\left(\lambda+\mu r^{2} / R_{0}{ }^{2}\right) \\
& \sigma_{r z}=G m \rho[\mu /(\lambda+2 \mu)]\left(r c / R_{0}{ }^{2}\right)
\end{aligned}
$$

where $R_{0}{ }^{2}=r^{2}+c^{2}$. We must remove these in order to make the surface $z=0$ stress free. We accomplish this by superposing two additional stress fields. The first is the field due to a line of force in the $z$ directon of intensity proportional to $B$ per unit length distributed uniformly along the negative $z$ axis between $-c$ and $-\infty$. Expressions for stress components are found by integrating the Kelvin solution [Timoshenko and Goodier, 1951, p. 354] for an isolated point force. We find for this line source that

$$
\begin{aligned}
\sigma_{z z} & =-B(1 / R)\left(\frac{2 \lambda+3 \mu}{\lambda+\mu}+\frac{z^{\prime 2}}{R^{2}}\right) \\
\sigma_{r z} & =-B(1 / r)\left(\frac{\lambda+2 \mu}{\lambda+\mu}-\frac{\mu z^{\prime}}{R(\lambda+\mu)}-\frac{z^{\prime 3}}{R^{3}}\right) \\
& \vee \\
\sigma_{\theta \theta}= & B \mu /(\lambda+\mu) R \\
\sigma_{r r} & =B(1 / R)\left(\frac{\mu}{\lambda+\mu}-\frac{r^{2}}{R^{2}}\right)
\end{aligned}
$$

The second field is due to a line of centers of dilatation along the negative $z$ axis, which increase in intensity linearly with $z$, from $-c$ to $-\infty$. The stress field due to an isolated center of dilation is given by Timoshenko and Goodier [1951, p. 362]. Integrating these, denoting the rate of increase in intensity by $A$, we find

$$
\begin{aligned}
& \sigma_{2 z}=-(A / 2 R)\left[1+\left(z^{\prime 2} / R^{2}\right)-\left(z z^{\prime} / R^{2}\right)\right] \\
& \sigma_{r z}=-(A / 2 r)\left[1-\left(z^{\prime} / R^{3}\right)-\left(r^{2} z / R^{3}\right)\right] \\
& \sigma_{\theta \theta}=(A / 2)\left[(1 / R)-\left(z / r^{2}\right)+\left(z z^{\prime} / r^{2} R\right)\right] \\
& \sigma_{r r}=(A / 2)\left[\left(c z^{\prime} / R^{9}\right)+\left(z / r^{2}\right)-\left(z z^{\prime} / r^{2} R\right)\right]
\end{aligned}
$$

Expressions for $\sigma_{z z}$ and for $\sigma_{r z}$ from (A5)-(A7) are summed, set equal to zero, and the resulting equations are solved for $A$ and $B$ :

$$
\begin{aligned}
& A=2 G m \rho \\
& B=-G m \rho(\lambda+\mu) /(\lambda+2 \mu)
\end{aligned}
$$

The final expressions for the stress components are found by introducing (A8) into the sums of the stress components in (A4), (A6), and (A7); the results are given by (7).

\section{Uplift Resulting From a Long Thrust Fault}

Surface uplift is calculated by applying the reciprocal theorem to solutions for a line force on a half-space. Consider a half-space loaded by a vertical line force $P$ per unit length on the surface, producing the stresses $\sigma_{l j}{ }^{*}$ at depth. Now consider an arbitrary surface $s$ in the half-space loaded such that the surface is displaced a distance $u$, and a point under the force $P$ is displaced a vertical distance $h$. Applying the reciprocal theorem to these two states gives

$$
P h=\int_{s} \sigma_{l j} u_{i} d s_{J}
$$

or

$$
h=\int_{s}\left(\sigma_{i} * / P\right) u_{i} d s_{j}
$$

Expressions for $\left(\sigma_{i j} * / P\right)$ needed for evaluating (A9) are given, for example, by Timoshenko and Goodier [1951, p. 85]; we find for the case where the surface $s$ is parallel to the $y$ axis, that the expressions are

$$
\begin{aligned}
& \sigma_{x x}{ }^{*} / P=(2 / \pi)\left(x^{2} z\right) /\left(x^{2}+z^{2}\right)^{2} \\
& \sigma_{z z}{ }^{*} / P=(2 / \pi)\left(z^{3}\right) /\left(x^{2}+z^{2}\right)^{2} \\
& \sigma_{x z}{ }^{*} / P=(2 / \pi)\left(x z^{2}\right) /\left(x^{2}+z^{2}\right)^{2}
\end{aligned}
$$


The shear stress $\sigma_{s n}{ }^{*}$ acting on an element of the surface $s$ dipping at an angle $\beta$ from the horizontal is therefore

$\left(\sigma_{s n}^{*} / P\right)=(z / \pi)[2 x z \cos 2 \beta$

$$
\left.-\left(x^{2}-z^{2}\right) \sin 2 \beta\right] /\left(x^{2}+z^{2}\right)^{2}
$$

Uplift at a point on the surface is found by evaluating (A9) using (A11). Note that the resulting integral is the same, except for a constant factor, as the integral giving the change in gravity at that point (see (20) and (21)).

Acknowledgments. One of us (J.B.W.) began this analysis while he was a visiting scientist with the U.S. Geological Survey; he was supported during later stages by W. F. Brace through the Cecil and Ida Green Professorship of Earth Sciences. J.R.R. was supported by the National Science Foundation and the U.S. Geological Survey. Discussions with several people, particularly, J. C. Savage and, recently, J. B. Rundle, were helpful during the course of the study. Also, we thank J. B. Rundle for pointing out a significant algebraic error in an earlier version of the analysis.

\section{REFERENCES}

Barnes, D. F., Gravity changes during the Alaskan earthquake, $J$. Geophys. Res., 7I, 451, 1966.

Chen, Y., H. Gu, and Z. Lu, Variations of gravity before and after the Heicheng earthquake, 1975, and the Tangshan earthquake, 1976, paper presented at Joint Assemblies of International Associations of
Seismology and Physics of the Earth's Interior, and Volcanology and Chemistry of the Earth's Interior, Durham, N. C., 1977.

Fung, Y. C., Foundations of Solid Mechanics, Prentice-Hall, Englewood Cliffs, N. J., 1965.

Hunt, T. M., Gravity changes associated with the 1968 Inangahua earthquake, N. Z. J. Geol. Geophys., 13, 1050, 1970.

Kisslinger, C., Processes during the Matsushiro, Japan, earthquake swarm as revealed by leveling, gravity, and spring-flow observations, Geology, 3, 57, 1975.

Nur, A., Matsushiro earthquake swarm: Confirmation of the dilatancy-diffusion model, Geology, 2, 217, 1974.

Oliver, H. W., S. L. Robbins, R. B. Grannell, R. W. Alewine, and S. Biehler, Surface and subsurface movements determined by remeasuring gravity, San Fernando Earthquake of February 9, 1971, Calif. Div. Mines Geol. Bull., 196, 1972.

Ruff, L. J., N. Goulty, J. Rial, J. Whitcomb, and C. Claflin, Monitoring of temporal gravity change in Southern California (abstract), Eos Trans. $A G U, 57(12), 898,1976$.

Rundle, J. B., Gravity changes and the Palmdale uplift, Geophys. Res. Lett., 5, 41-44, 1978.

Stuart, W. D., and M. J. S. Johnston, Intrusive origin of the Matsushiro earthquake swarm, Geology, 3, 63, 1975.

Timoshenko, S., and J. N. Goodier, Theory of Elasticity, McGrawHill, New York, 1951

Whitcomb, J. H., New vertical geodesy, J. Geophys. Res., 8I(26), 4937-4944, 1976.

(Received February 7, 1978;

revised August 29, 1978;

accepted September 7, 1978.) 